Short title: OsG1L1 and OsG1L2 regulate inflorescence branching in rice

\title{
The ALOG family members OsG1L1 and OsG1L2 regulate inflorescence branching in rice
}

4

Emanuela Franchini²,a , Veronica M. Beretta ${ }^{2, a}$, Israr Ud Din²a,b, Elia Lacchini ${ }^{a, c}$, Lisa Van den Broeck $^{d}$, Rosangela Sozzani ${ }^{d}$, Gregorio Orozco-Arroyo ${ }^{a}$, Hélène Adam ${ }^{e}$, Stefan Jouannic ${ }^{e}$, Veronica Gregis ${ }^{\mathrm{a}}$ and Martin M. Kater ${ }^{\mathrm{a}, 3}$

Affiliations:

a Dipartimento di Bioscienze, Università degli Studi di Milano, Via Celoria 26, 20133, Milano, Italy

${ }^{b}$ Present address: Institute of Biotechnology and Genetic Engineering (IBGE),

University of Agriculture, Peshawar 25130, Pakistan

'Present address: VIB Center for Plant Systems Biology, Technologiepark 71, B-9052 Ghent,

Belgium

d Plant and Microbial Biology Department, North Carolina State University, Raleigh, NC 27695

e DIADE, University of Montpellier, IRD, CIRAD, Montpellier, France

${ }^{1}$ This work was supported by MUR PRIN2017_2017N5LBZK

The PhD fellowship of E.F. and V.M.B, were supported by the Doctorate School in Molecular and Cellular Biology, Università degli Studi di Milano. E.F. was supported by H2020-MSCA-RISE-2015 ExpoSEED Proposal Number: 691109.

${ }^{2}$ These authors contributed equally to the article.

${ }^{3}$ Corresponding author: martin.kater@unimi.it

ORCID IDs: 0000-0003-3293-5137 (V.M.B.), 0000-0002-0643-2408 (I.U.D.), 0000-0002-1598-8950 (E.L.), 0000-0001-7509-6244 (G.O.A.), 0000-0003-0226-0757 (L.V.B.), 0000-0003-3316-2367 (R.S.), 0000-0003-0324-6201 (H.A.), 0000-0001-5777-3336 (S.J.), 0000-0003-1876-9849 (V.G.), 0000-0003-1155-2575 (M.K.)

One-sentence summary: OsG1L1 and OsG1L2 control panicle architecture through delaying the transition from indeterminate branch- to determinate spikelet-meristem identity.

Author contributions: V.G. and M.K.conceived and designed the research. E.F., V.M.B., I.U.D., G.O.A., L.V.B., R.S. and E.L. carried out and interpreted the experiments. H.A. and S.J. contributed to experiment design and analysis. E.F., V.M.B. and M.K. wrote the article with the contribution of all the authors.

\section{ABSTRACT}

The architecture of the rice inflorescence is an important determinant of seed yield. The length of the inflorescence and the number of branches are among the key factors determining the amount of 
spikelets, and thus seeds, that will develop. Especially the timing of the identity transition from indeterminate branch meristem to determinate spikelet meristem regulates the complexity of the inflorescence. In this context, the ALOG gene TAWAWA1 (TAW1) has been shown to delay the transition to determinate spikelet development in rice. Recently, by combining precise laser microdissection of inflorescence meristems with RNA-seq we observed that two ALOG genes, Oryza sativa OsG1-like 1 (OsG1L1) and OsG1L2, have an expression profile similar to TAW1. Here we report that osg $1 / 1$ and osg $1 / 2$ loss-of-function CRISPR mutants have similar phenotypes as the taw 1 mutant, suggesting that these genes might act on related pathways during inflorescence development. Transcriptome analysis of the osg1/2 mutant suggested interactions of OsG1L2 with other known inflorescence architecture regulators and the datasets were also used for the construction of a gene regulatory network (GRN) proposing interactions between genes potentially involved in controlling inflorescence development in rice.

The spatio-temporal expression profiling and phenotypical analysis of CRISPR loss-of-function mutants of the homeodomain-leucine zipper transcription factor gene OsHOX14 suggest that the proposed GRN indeed serves as a valuable resource for the identification of new players involved in rice inflorescence development.

\section{INTRODUCTION}

The inflorescences of land plants show a wealth of distinct architectures which evidences its importance for their reproductive success. The plant species or family-specific inflorescence shape depends on the activity and identity of meristems which determine the degree of branching and the number of flowers that will ultimately develop. Inflorescence meristems are defined as indeterminate since they continue to develop meristems in the axils of lateral organs, such as bracts. On the contrary, floral meristems are determinate as the development of the floral organs exhausts meristematic activity. In this sense, the formation of flowers can be seen as a developmental endpoint. An extreme example are tulips, where the apical meristem transforms into a floral meristem and forms one single apical flower. It is thus the identity transitions from indeterminate to determinate meristems that defines the complexity of an inflorescence (Hake, 2008).

Oryza sativa, commonly known as rice, develops a complex and determinate inflorescence, named panicle (Bommert et al., 2005; Han et al., 2014). Its architecture is established during early stages of rice reproductive development and it depends on the activity of different meristem types (Tanaka et al., 2013; Caselli et al., 2020). During the floral transition, the rice Shoot Apical Meristem (SAM) becomes Inflorescence Meristem (IM), also called rachis meristem. The IM gives rise to Primary Branch Meristems (PBMs) that produce Axillary meristems (AMs) which could differentiate into indeterminate Secondary Branch Meristems (SBMs) or determinate Spikelet Meristems (SMs). In 
the same way SBMs elongate and produce SMs. In rice, the SM develops three floral meristems (FMs), of which one will differentiate into one fertile floret, whereas the other two will develop into empty glumes (sterile lemmas); thus exhausting the pool of meristematic cells (Bommert et al., 2005; Han et al. 2014). Furthermore, the length of the rice inflorescence, and consequently the number of primary branches that can develop, is also determined by the timing of IM abortion.

Rice plants in which early transitions to spikelet meristem identity occur will have less complex panicles with fewer seeds in contrast to plants in which the transition is delayed. Among the genes that have been identified to control this transition are ABERRANT PANICLE ORGANIZATION1 (APO1) and APO2 (Ikeda-Kawakatsu et al., 2012). Both are mainly expressed in IM and in BMs, where they also promote cellular proliferation. They are orthologs of the Arabidopsis thaliana genes UNUSUAL FLORAL ORGANS (UFO) and LEAFY (LFY), respectively. However, while the UFO and $L F Y$ promote floral identity, $A P O 1$ and $A P O 2$ repress the transition to determinate spikelet meristem formation (Ikeda-Kawakatsu, et al., 2009; Ikeda-Kawakatsu, et al., 2012). Recently, it was shown that LARGE2, a HECT-domain E3 ubiquitin ligase OsUPL2, interacts directly with APO1 and APO2 to modulate their stability. Genetic analysis of the large2 mutant, which displays bigger panicles with more branches and seeds, confirmed that LARGE2 functions in a common pathway with APO1 and APO2 (Huang et al., 2021).

TAWAWA1 (TAW1)/G1-LIKE 5 (G1L5) is another gene that promotes BM identity and suppresses SM specification by activating genes involved in the repression of floral transition (Yoshida et al., 2013). The dominant taw1-D gain-of-function mutant shows a delay in spikelet specification which results in increased branching and higher seed numbers. TAW1 belongs to the Arabidopsis $L S H 1$ and Oryza G1 (ALOG) gene family, which includes fourteen ALOG genes in rice. The ALOG domain is highly conserved among land plants and evolutionary studies propose that it derived from the $\mathrm{N}$ terminal DNA-binding domain of integrases that belong to the tyrosine recombinase superfamily which are encoded by a distinct type of DIRS1-like LTR retrotransposons that are found in several eukaryotes (Lyer \& Aravind, 2012).

Recently, Harrop et al. (2016) used laser microdissection microscopy to specifically isolate different rice inflorescence meristem tissues for RNA-seq based expression profiling. Subsequent transcriptome analysis revealed that two ALOG genes, OsG1L1 and OsG1L2, have similar expression profiles as TAW1. All three genes are highly expressed in the IM and subsequently, their expression gradually decreases in PBM, ePBM/AMs and SM. Phylogenetic analysis showed that OsG1L1 and OsG1L2 cluster both in subgroup A and are therefore more distantly related to TAW1 which belongs to subgroup C (Li et al., 2019).

Here, we describe the functional analysis of OsG1L1 and OsG1L2 which evidenced that both genes play a similar role in rice inflorescence development. Like in the taw1-3 and TAW1 RNAi lines, both osg1/1 and osg1/2 CRISPR-Cas9 mutants showed a reduced branching phenotype and lower seed numbers. RNA-seq analysis of developing g1/2 inflorescences was used to compute a gene 
regulatory network (GRN). Validation of the resulting network by a preliminary functional study of the homeodomain-leucine zipper transcription factor gene OsHOX14 suggests that the proposed GRN could serve as a valid resource for the identification of genes controlling inflorescence development in rice.

\section{RESULTS}

\section{OsG1L1 and OsG1L2 expression analysis}

127 Previous transcriptome analysis of laser micro-dissected rice reproductive meristems, allowed identification of two ALOG genes, OsG1L1 and OsG1L2 that share a similar expression profile with the previously studied TAW1 gene (Harrop et al., 2016; Yoshida et al. 2013; Figure 1A). These genes are highly expressed in the Inflorescence Meristem (IM), then mRNA abundance decreases in the Primary Branch Meristem (PBM), and a further reduction is observed in the elongated PBM with axillary meristem (ePBM/AM). In the Spikelet Meristem (SM) there is a slight increase in mRNA levels for all three genes.

To determine in more detail the expression pattern of OsG1L1 and OsG1L2 and to confirm their expression in inflorescence meristems, we performed real-time PCR on different plant tissues such as, the root, the root tip (where the Root Apical Meristem (RAM) is localized), young and mature leaves, the Shoot Apical Meristem (SAM) and all reproductive meristems enriched tissues, like IM, PBM, ePBM/AM and SM/FM, and milk and mature seeds, respectively at 8 and 30 days after fertilization. Since the expression of TAW1 was already described previously (Yoshida et al., 2013), it was used as a positive control. This analysis showed that all three genes are preferentially expressed in inflorescence tissues (IM, PBM, ePBM/AM and SM/FM) (Figure 1B). RNA in situ hybridisation was performed to further investigate and compare the spatiotemporal expression of OsG1L1, OsG1L2 and TAW1 during different stages of panicle development. We designed for each of the three genes a specific digoxigenin-labelled RNA probe. This analysis revealed that OsG1L1 and OsG1L2 have a similar expression profile as TAW1. All three genes are expressed in the IM, PBM, ePBM/AM and SM/FM, suggesting OsG1L1 and OsG1L2 might have a similar functional role in the inflorescence meristems as TAW1 (Figure 1C). 
A

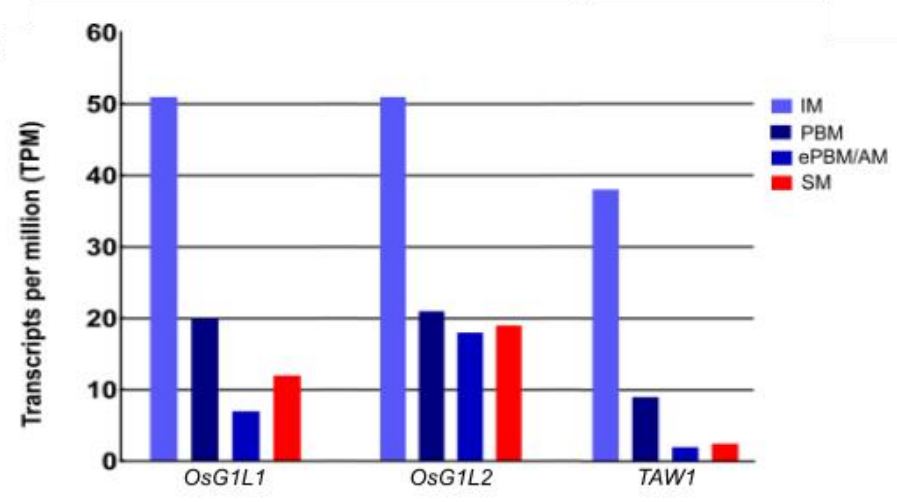

$\mathrm{B}$
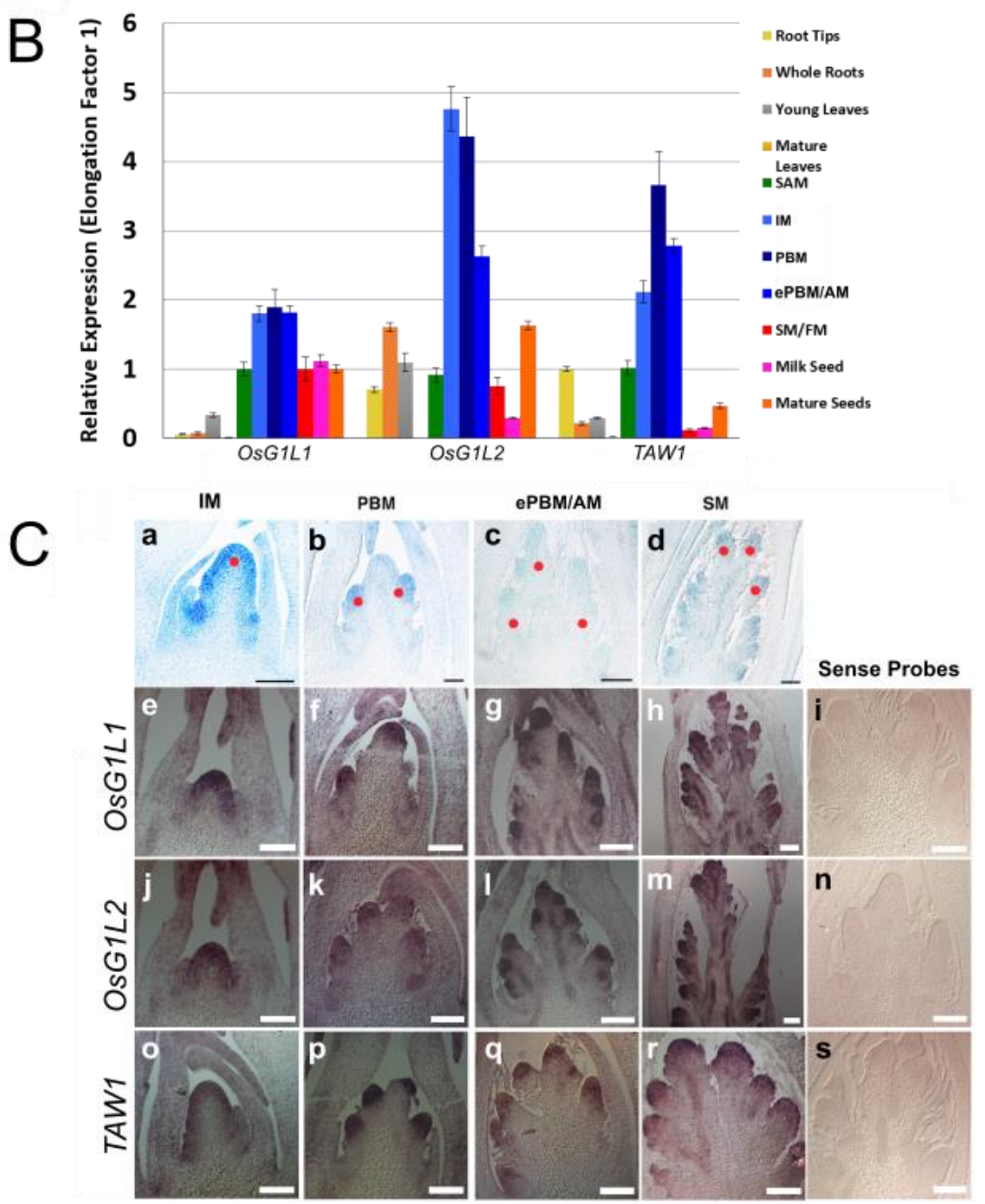

Figure 1. The ALOG family genes OsG1L1, OsG1L2 and TAW1 are highly expressed in inflorescence meristems. A, four reproductive meristem types sampled and analysed by RNA-seq (Harrop et al. 2016). Read counts are expressed in transcripts per million. B, qRT-PCR of OsG1L1, OsG1L2 and TAW1 across different tissues: vegetative tissues (roots and leaves), reproductive meristems enriched tissues manually dissected (IM, PBM, ePBM/AM, SM/FM) and seeds. C, Expression pattern of OsG1L1, OsG1L2 and TAW1 analysed by in situ hybridization at four developmental stages. Representation of four developmental stages that were analysed with reference to the meristem types indicated above the figure (a-d), where red dots indicate respectively the different meristematic tissues: IM (a), PBM (b), ePBM/AM (c) and SM (d). OsG1L1 Antisense 
control probe (s). Scale bars represent $50 \mu \mathrm{m}(\mathrm{a}-\mathrm{c})$ and $100 \mu \mathrm{m}$ (d-s). [Inflorescence Meristem (IM), Primary Branch Meristem (PBM), elongated PBM with Axillary Meristem (ePBM/AM), Spikelet Meristem (SM), Flower Meristem (FM)].

\section{Analysis of the osg1/1 and osg1/2 mutant phenotypes}

To functionally characterize OsG1L1 and OsG1L2 CRISPR-Cas9 genome editing technology was used to generate mutations in these genes. A specific single-guide RNA (sgRNA) was designed for each gene and the two CRISPR-constructs (Miao et al. 2013) were used for Agrobacterium tumefaciens mediated transformation of rice embryonic calli. The two sgRNAs were designed to create indels in the ALOG domain.

The sgRNA designed for OsG1L1 targeted the first exon at $397 \mathrm{bp}$ from the ATG start site, whereas the sgRNA for OsG1L2 was designed to target a region 131 bp downstream from the ATG start site (Supplemental Figure S1 A; Supplemental Figure S2 A). For OsG1L1, five T0 transgenic plants with different mutations at the sgRNA target site were obtained. Some of these mutations were in frame ( 3 and 6 bp deletions) and were not further analysed. Two independent transformants had a homozygous deletion of $2 \mathrm{bp}(\mathrm{AG})$ at $145 \mathrm{bp}$ from the translation start site. This mutation created a frameshift resulting in the formation of an aberrant protein, characterized by the disruption of the ALOG domain and of the putative nuclear localization signal (NLS). For these reasons, the obtained aberrant protein is most likely not functional (Supplemental Figure S1 B-C). We used for further analysis these two osg1/1 mutants and in the T2 generation we obtained homozygous plants without the Cas9 encoding T-DNA insertion.

For the CRISPR-construct targeting OsG1L2, eighteen T0 transgenic rice plants were generated. All these plants had a similar frameshift mutation due to the insertion of a single base pair (A, C, G or $\mathrm{T})$ at $148 \mathrm{bp}$ from the start site. The $\mathrm{A}$ insertion leads to the formation of a premature stop codon (TGA), resulting in the production of a truncated protein of 49 amino acids (Supplemental Figure S2 $B-C)$, lacking the ALOG domain and the putative NLS. The insertion of one of the other bases (C, G, T) leads to the formation of an out of frame reading frame resulting in a protein of 176 amino acids without the ALOG domain and the putative NLS (Supplemental Figure S2 C). We selected in the T1 generation two independent osg1/2 mutant lines having an $\mathrm{A}$ or $\mathrm{C}$ insertion. In the $\mathrm{T} 2$ generation we obtained lines homozygous for these insertions and without the Cas9 encoding T-DNA insertion. For detailed phenotyping we used the line with the A insertion.

During the vegetative growth, the osg1/1 and osg1/2 mutants didn't show any obvious phenotype (data not shown). Considering that the expression of OsG1L1 and OsG1L2 was predominantly restricted to inflorescence meristem tissues, a detailed phenotypic analysis of the panicle was performed using Panicle TRAit Phenotyping software (P-TRAP) (Figure 2; AL-Tam et al., 2013). To obtain a robust statistical analysis, at least 15 plants for each genotype were analysed. In particular we analysed 15 wild-type plants, 19 osg1/1 mutants and 20 osg1/2 mutants. After panicle imaging, 
198 the P-TRAP software quantified the traits related to the panicle architecture and seed numbers 199 (Supplemental Table S1).

200 This analysis showed that osg1/1 and osg1/2 produced significantly shorter panicles than wild-type 201 plants. Furthermore, their panicles developed fewer PBs, SBs and spikelets/seeds. In particular, the 202 osg1/1 mutants produced panicles that were on average $2 \mathrm{~cm}$ shorter than wild type and developed 203 on average 3 PB and 30 spikelets less than wild-type plants. Interestingly, the number of SBs were 204 not significantly different from wild type (Figure $2 \mathrm{C}-\mathrm{F}$ ).

205 The osg1/2 mutant plants produced panicles that were on average $1.5 \mathrm{~cm}$ shorter than wild type and 206 developed on average 4 PBs, 8 SBs and 51 spikelets/seeds less than wild-type plants (Figure 2 C207 F). This analysis confirmed a previous independent experiment in which PBs, SBs and spikelet/seed 208 numbers were compared between wild-type and osg1/2 plants with a different mutation (a $\mathrm{C}$ insertion 209 at $148 \mathrm{bp}$ from the ATG) (Supplemental Figure S3).

210 The P-TRAP analysis revealed also that both osg1/1 and osg1/2 mutant lines had longer PBs and 211 SBs as well as longer internodes in PBs (Figure $2 \mathrm{G}-\mathrm{I}$ ). In detail, the osg1/1 mutant plants displayed 212 PBs and SBs that were on average respectively $1 \mathrm{~cm}$ and $0.5 \mathrm{~cm}$ longer than those of wild-type rice 213 plants. The internodes of PBs were on average $0.4 \mathrm{~cm}$ longer than in wild type. The osg $1 / 2$ mutant 214 plants instead produced PBs and SBs that were on average $1.6 \mathrm{~cm}$ and $1 \mathrm{~cm}$ longer than wild type, 215 respectively. The internodes of PBs were on average $0.7 \mathrm{~cm}$ longer than wild type. Overall, both 216 mutants showed similar aberrations in panicle architecture although the phenotype of the osg1/2 217 mutant was more severe. 
A

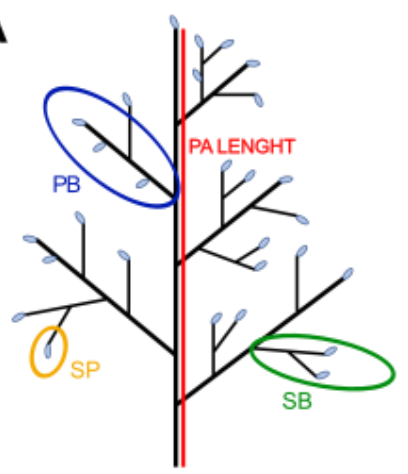

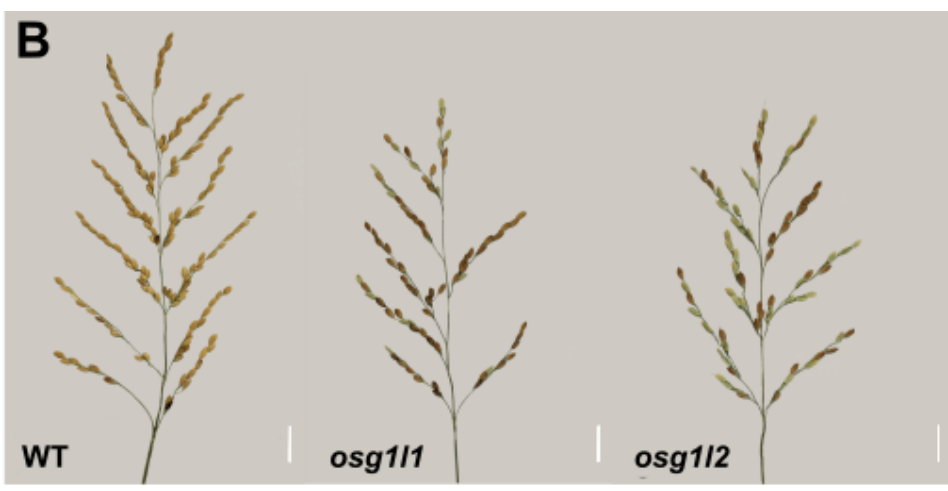

D
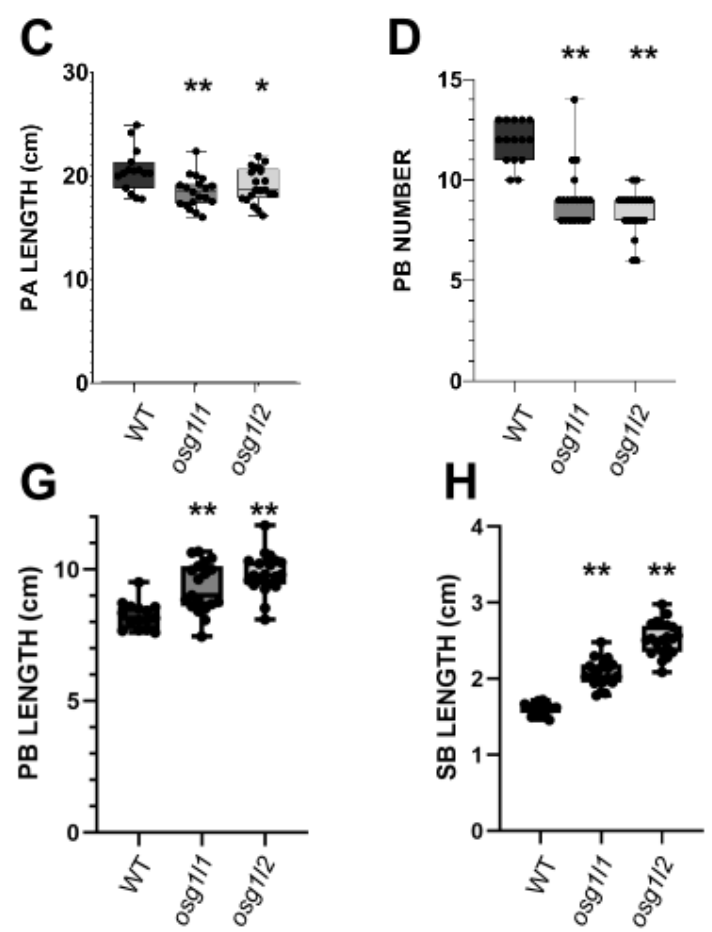

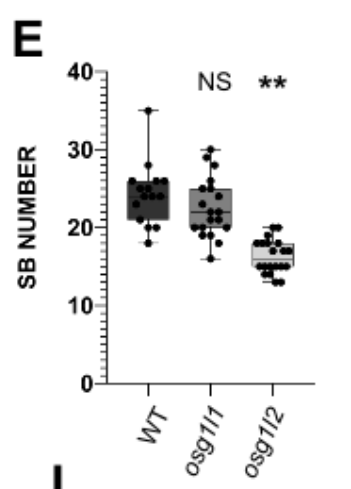

F
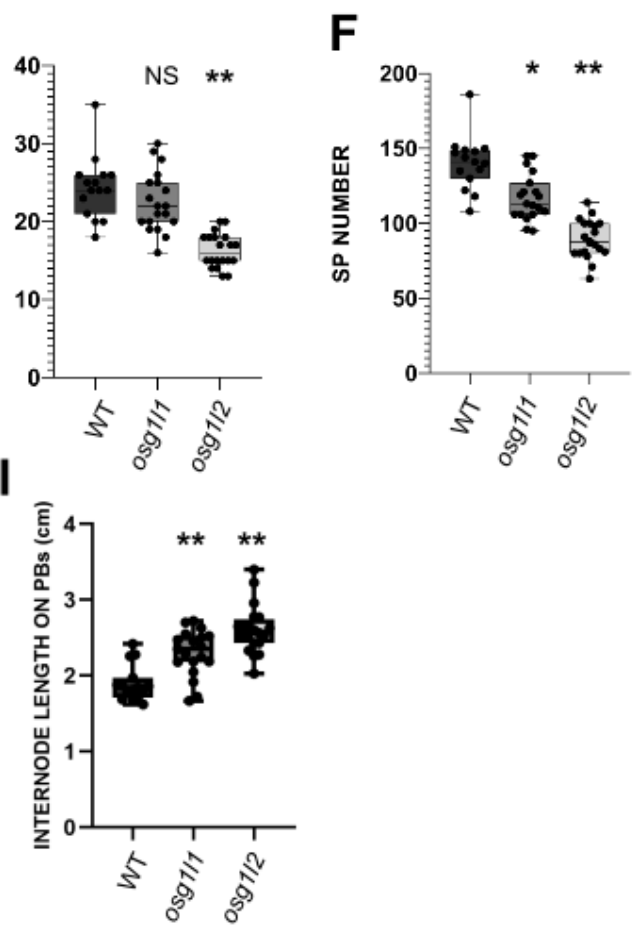

Figure 2. Phenotypical analysis of panicle architecture in wild type, osg1/1 and osg1/2 mutants. $A$, Schematic representation of panicle structure $[P A=$ panicle; $P B=$ primary branches; $\mathrm{SB}=$ secondary branches; $\mathrm{SP}=$ spikelets/seeds]. B, Main panicles of WT, osg1/1 and osg $1 / 2(2 \mathrm{~cm}$ scale bars). Graphs representing the comparison of: (C) Panicle (PA) Length, (D) Primary Branch (PB) number, (E) Secondary Branch (SB) number, (F) Spikelet/seed (SP) number in WT, osg1/1 and osg1/2 backgrounds. Graphical representation of the comparison between the: $(G)$ length of Primary Branches (PB), (H) length of Secondary branches (SB) and (I) length of the internodes in PBs. OneWay ANOVA with Tukey test; ${ }^{* *} p<0,01 ;{ }^{*} p<0,05$.

To investigate whether loss-of-function of OsG1L1 and OsG1L2 not only affected seed numbers but also seed size, the length, width and surface area of osg1/1 and osg1/2 mutant seeds were measured. For each genotype (osg1/1, osg1/2 and wild type) a minimum of 100 seeds were analysed using the Smart Grain software (Tanabata et al., 2012; Supplemental Table S2). This analysis revealed that osg1/1 and osg1/2 mutants produced significantly smaller seeds than wild type. Both 
osg1/1 and osg1/2 seeds had smaller seed areas, where osg1/1 seeds had a reduced length and width and osg1/2 seeds only showed a reduction in seed width compared to wild type (Figure 3).
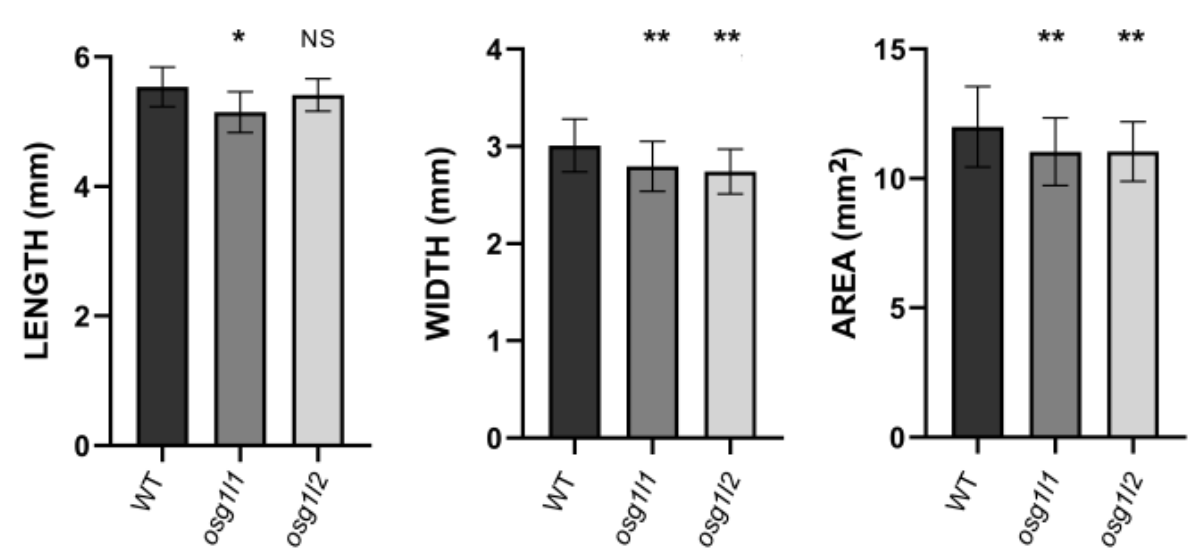

Figure 3. Size measurements of wild type, osg1/1 and osg1/2 seeds.

Graphs resulting from the analysis of length, width and area of wild type (WT), osg1/1 and osg1/2 seeds using the Smart Grain software (Tanabata et al., 2012). 100 seeds for each genotype were analysed. Statistical One-Way ANOVA with Tukey test: ${ }^{* *} \mathrm{p}<0,01 ;{ }^{*} \mathrm{p}<0,05$. NS $=$ not significant.

\section{Transcriptome analysis of the osg1/2 mutant at early stages of inflorescence} development

The phenotypic analysis of the osg1/1 and osg1/2 mutants suggests that both genes may play a similar role during rice inflorescence development. Since inflorescence branching was more severely affected in the osg1/2 mutant, this line was selected for RNA-seq transcriptome analysis to obtain deeper insights into the role this $A L O G$ gene plays at the early stages of inflorescence development. Developing inflorescences of the wild type and the osg1/2 mutant at early developmental stages enriched in PBMs and ePBM/AMs were manually dissected. Material for four biological replicates was obtained, each replicate consisting of 8 to 10 dissected inflorescences. Subsequently, RNA was extracted and used for Illumina sequencing.

The raw RNA-seq files were processed using the TuxNet interface (Spurney et al., 2019). Reads were cleaned, mapped on the $O$. sativa reference genome (IRGSP-1.0), normalized, and FPKMs (Fragment per kilobase of transcript per million mapped reads) were calculated (Supplemental Table S3). Finally, performing a pairwise differential expression analysis between the wild type and mutant, the TuxNet interface generated Differentially Expressed Gene (DEG) datasets (Spurney et al., 2019) (Supplemental Table S4). After data processing, an average of approximately 13.000 .000 reads for each replicate were obtained. The alignment of the reads to the reference genome resulted overall in $>97 \%$ coverage. Setting the $\log _{2}$ (Fold Change $(F C)$ ) equal to 1 and the q-value equal to 0.05 , a total of 279 differentially expressed genes were identified, of which 152 were downregulated and 127 upregulated in the osg1/2 mutant compared to the wild type. Among the upregulated genes in the 
osg112 mutant, we identified OsMADS37, a MADS-box transcription factor encoding gene homologous to Arabidopsis FLOWERING LOCUS C (FLC) (Ruelens et al., 2013), OsERF112, an ERF/AP2 transcription factor (Nakano et al., 2006), and OsG1L4, another member of the ALOG gene family. Interestingly, also EPIGENETIC SHORT PANICLE (OSESP), a putative long-noncoding RNA whose overexpression leads to shorter and denser panicles, was upregulated in the osg1/2 mutant (Luan et al., 2019). Moreover, the expression of genes involved in hormonal pathways was upregulated, such as OsRR3, an A-type response regulator that acts as a negative regulator of cytokinin signalling (Cheng et al., 2010). Among the upregulated genes there are several which encode for Zinc-finger transporter proteins and genes encoding for proteins containing a NB-ARC domain, which is associated to plant disease resistance (Van Ooijen et al., 2008) (Supplemental 275 Table S4 and Table 1).

276 Among the downregulated genes, we found TFs like ONAC120 that is similar to NAM/CUC-2 like protein (Ooka et al., 2003) and two genes belonging to the TCP family, OsTCP25 and OsTB1/FC1 (that henceforth will be referred to as OsFC1). Interestingly, OsFC1 is already known to be a negative regulator of tillering and inflorescence development (Takeda et al., 2003; Cui et al., 2020). Notably, the transcription factors OsMADS34/PAP2 and OsGATA7, which are known to be involved in inflorescence architecture establishment; and OsHOX14, which has been proposed to be involved 282 in panicle development, were also downregulated (Gao et al. 2010; Kobayashi et al., 2012; Shao et 283 al., 2018; Zhang et al., 2018). Additional downregulated genes that have been associated with 284 inflorescence development are OsPP2C1 (Protein phosphatase 2C), OsRCN1 and OsRCN4 285 (Putative phosphatidylethanolamine-binding protein and Rice TFL1/CEN homolog), and OsCEP6 286 (C-terminally encoded peptide) (Nakagawa et al., 2002; Li et al., 2013; Sui et al., 2016). Moreover, we could also find genes involved in hormonal pathways like OsIAA14, belonging to the Aux/IAA family and involved in auxin-response (Jain et al., 2006) (Table 1 and Supplemental Table S4). 


\begin{tabular}{|c|c|c|c|c|}
\hline Gene name & Gene ID & $\log 2$ FC & Q-value & Putative function \\
\hline OsESP & Os01g0356951 & $6,55 \mathrm{E}+04$ & 0,00245724 & $\begin{array}{l}\text { Involved in the regulation of } \\
\text { panicle architecture }\end{array}$ \\
\hline OsMADS37 & Os08g0531900 & $1,87 \mathrm{E}+00$ & 0,00245724 & Homolog of $F L C$ in rice \\
\hline OsG1L4 & Os04g0516200 & $1,34 \mathrm{E}+00$ & 0,00245724 & $\begin{array}{l}\text { Protein of unknown function } \\
\text { DUF640 domain containing } \\
\text { protein }\end{array}$ \\
\hline OsERF112 & Os12g0603300 & $1,09 \mathrm{E}+00$ & 0,00245724 & $\begin{array}{l}\text { Similar to AP2 domain containing } \\
\text { protein }\end{array}$ \\
\hline OSRR3 & Os02g0830200 & $1,95 \mathrm{E}+00$ & 0,00245724 & $\begin{array}{l}\text { A-type response regulator } \\
\text { involved in Cytokinin signaling }\end{array}$ \\
\hline ONAC120 & Os10g0477600 & $-2,47 \mathrm{E}+00$ & 0,00245724 & $\begin{array}{l}\text { Similar to NAM / CUC2-like } \\
\text { protein }\end{array}$ \\
\hline OsTCP25 & Os09g0521300 & $-1,07 E+00$ & 0,0485909 & $\begin{array}{l}\text { Similar to } T C P \text { family transcription } \\
\text { factor }\end{array}$ \\
\hline OsTB1 & Os03g0706500 & $-1,12 \mathrm{E}+00$ & 0,00245724 & $\begin{array}{l}T C P \text { family transcription factor, } \\
\text { negative regulator of lateral } \\
\text { branching }\end{array}$ \\
\hline OsPP2C30 & Os03g0268600 & $-1,01 E+00$ & 0,00245724 & $\begin{array}{l}\text { Similar to Protein phosphatase } \\
\text { type } 2 \mathrm{C}\end{array}$ \\
\hline OsMADS34/PAP2 & Os03g0753100 & $-1,13 E+00$ & 0,00245724 & $\begin{array}{l}\text { MADS-box transcription factor, } \\
\text { involved in inflorescence and } \\
\text { spikelet development }\end{array}$ \\
\hline OsRCN1 & Os11g0152500 & $-1,04 E+00$ & 0,00245724 & $\begin{array}{l}\text { Putative } \\
\text { phosphatidylethanolamine-binding } \\
\text { protein, Rice } T F L 1 / C E N \text { homolog. } \\
\text { Involved in the control of } \\
\text { inflorescence architecture and in } \\
\text { the repression of flowering }\end{array}$ \\
\hline OsRCN4 & Os04g0411400 & $-1,48 E+00$ & 0,00839011 & Terminal flower 1 -like protein \\
\hline OsHOX14 & Os07g0581700 & $-1,53 E+00$ & 0,00456853 & $\begin{array}{l}\text { Homeodomain-leucine zipper } \\
\text { (HD-Zip) transcription factor, may } \\
\text { be involved in the regulation of } \\
\text { panicle development }\end{array}$ \\
\hline OsCEP6 & Os08g0475500 & $-1,02 \mathrm{E}+00$ & 0,00245724 & $\begin{array}{l}\text { C-terminally encoded peptide, } \\
\text { involved in the regulation of } \\
\text { panicle and grain development }\end{array}$ \\
\hline OsPP2C1 & Os09g0325700 & $-2,08 E+00$ & 0,00245724 & $\begin{array}{l}\text { Protein phosphatase } 2 \mathrm{C} \text {. Involved } \\
\text { in abiotic stress response and } \\
\text { early panicle development }\end{array}$ \\
\hline OsGATA7 & Os10g0557600 & $-1,49 \mathrm{E}+00$ & 0,00245724 & $\begin{array}{l}\text { GATA transcription factor. } \\
\text { Involved in brassinosteroids- } \\
\text { mediated growth regulation, } \\
\text { panicle development and grain } \\
\text { shape/number/weight/yield }\end{array}$ \\
\hline OsIAA14 & Os03g0797800 & $-1,44 \mathrm{E}+00$ & 0,00245724 & $\begin{array}{l}\text { Protein belonging to the AUX/IAA } \\
\text { protein family }\end{array}$ \\
\hline
\end{tabular}

Table 1. Genes differentially expressed in the osg1/2 mutant and involved in inflorescence development. For each gene is indicated the Gene Name (column one); the Gene ID (column two); the log2 Fold Change (log2 FC, column three); the Q-value (column four; a significance cut-off of 0.05 is applied) and information related to their function (column 5).

\section{Gene regulatory network inference predicts a functional role for OsHOX14}

296 To identify major regulatory transcription factors underlying the inflorescence phenotype of osg 1/2, 297 we built a gene regulatory network (GRN) using a regression tree with random forest approach 298 (Spurney et al., 2019). Specifically, we inferred causal relations between 15 identified differentially 299 expressed transcription factors (TFs) $\left(\log _{2}(F C)>1\right.$ or $<-1$, q-value $\left.<0.05\right)$ and 232 downstream genes in osg1/2 mutant $\left(\log _{2}(\mathrm{FC})>1\right.$ or $<-1$, q-value $\left.<0.05\right)$. The inferred network contained 79 
genes, of which five TFs have more than 10 outgoing regulations, including OsWRKY80 (Wu et al., 2004), OsG1L2, OsMADS37 (Ruelens et al., 2013), OsFC1 (Takeda et al., 2003; Cui et al., 2020), and OsGATA7 (Zhang et al., 2018) (Figure 4). One of these major regulators is OsG1L2, regulating 16 downstream genes, several of which have been shown to be involved in rice inflorescence development (Figure $4 \mathrm{C}$ ). For example, OsESP is a putative long-noncoding RNA whose gain-offunction mutant leads to a short and denser panicle (Luan et al., 2019) and OsJAZ4, also known as OsTIFY11b, is a positive regulator of grain-size acting downstream of TRIANGULAR HULL1 (OsTH1), another ALOG factor (Hakata et al., 2012; Wang et al., 2019). Interestingly, thirteen of the predicted OsG1L2 targets are upregulated in the osg1/2 mutant, ten of which are predicted to be coregulated by OsFC1, a gene known to negatively regulate branching (Takeda et al., 2003; Cui et al., 311 2020).

312 To identify regulatory subclusters within the osg1/2 network, we clustered the network genes into 313 different modules with the Cytoscape plugin clusterMaker2 (see materials and methods) (Figure 4). 314 A total of four modules were identified, one smaller module of 10 genes (Figure $4 \mathrm{~B}$ ), and three larger modules of 24, 23 and 22 genes (Figure 4 A, C, D) . The smaller module contains CYTOKININ-

316 RESPONSIVE GATA TRANSCRIPTION FACTOR1 (OSCGA1), of which constitutive 317 overexpression reduced grain filling (Hudson et al., 2013) and OsMADS37, the closest homologues 318 of Arabidopsis FLOWERING LOCUS C (Shrestha et al., 2014) (Figure $4 \mathrm{~B}$ ). One of the largest 319 modules contains two GATA TFs, including OsGATA7, which influences architecture and grain 320 shape and of which CRISPR/Cas9 lines show a similar phenotype as osg1/2 (Zhang et al., 2018) 321 (Figure $4 \mathrm{D}$ ). As we were interested in the regulatory interactions underlying the osg 112 mutant 322 phenotype, we focused on module C, which contains four TFs: OsG1L2, OsFC1, OsMADS34, and OsHOX14. Interestingly, OsMADS34 has been shown to be necessary for correct inflorescence development, further emphasizing the functional importance of this regulatory module (Kobayashi et al., 2012). OsHOX14 can form heterodimers with OsHOX12, a gene that regulates panicle exertion (Gao et al., 2016). Overall, our network analysis allowed for the identification of many interesting candidates, of which several have been described in the context of panicle development, and indicated putative targets of OsG1L2 and other major TFs, giving also hints of which genes might be involved in the same developmental pathway regulating a similar set of genes. 


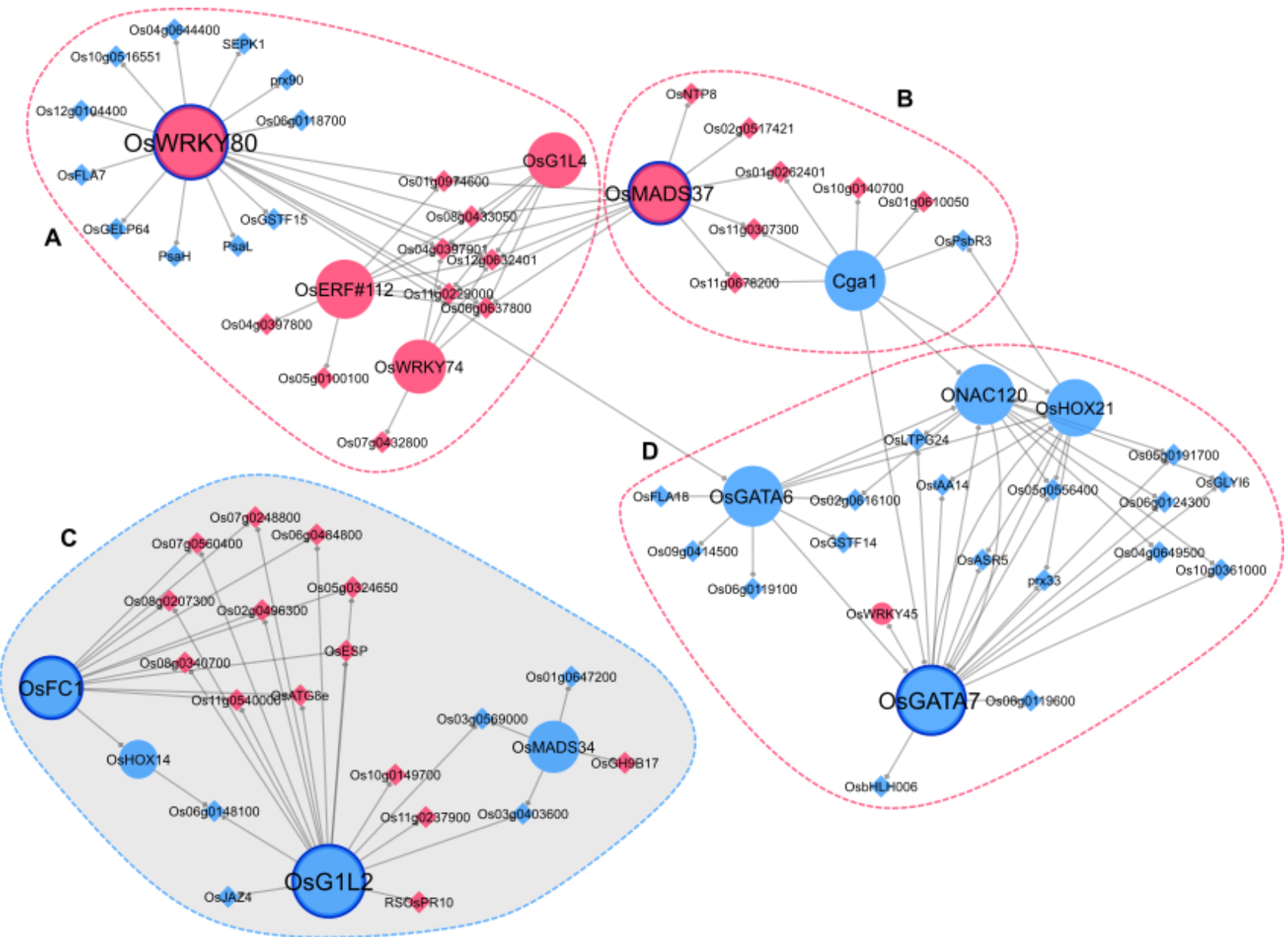

Figure 4. Graphic representation of the predicted Gene regulatory Network (GRN). Regulatory interactions were inferred using a regression tree with random forest approach. Transcription factors and other genes are represented in circles and diamonds, respectively. The interactions are represented by a diamond arrow. Upregulated and downregulated genes are highlighted in magenta and light blue respectively. Circled with a dotted line, there are the four major subclusters (A to D) in which the network can be divided. Circled in blue with a continuous line there are the five major TFs of the GRN: OsWRKY80, OsG1L2, OsMADS37, OsFC1, and OsGATA7. The module containing OsG1L2 is highlighted in grey $(\mathrm{C})$.

\section{Validation of GRNs by qRT-PCR}

RT-qPCR experiments were carried out to validate some of the deregulated genes of subcluster C of the predicted GRN (Figure 4). In particular, we focused on those genes that were already proposed to be involved in inflorescence development, such as OsHOX14, OsMADS34, OsFC1 and OsESP (Takeda et al., 2003; Gao et al. 2010; Shao et al., 2018; Luan et al., 2019). Os03g0569000 was also analysed because, according to the GRN, it was a common target of OsMADS34 and OsG1L2. The qRT-PCR was performed using three biological replicates of developing inflorescences enriched in PBMs and ePBM/AMs of wild-type and the osg1/2 mutant. Furthermore, the expression of the selected genes was also analysed in the osg1/1 mutant, to determine if these genes might be regulated by both $A L O G$ genes. 
As shown in Figure 5 (A-E), the qRT-PCR confirmed the downregulation of OsHOX14, OsMADS34, OsFC1 and Os03g0569000 and the upregulation of OsESP in osg1/2 mutant compared to WT.

The expression analysis performed on the selected genes in the osg1/1 mutant highlighted that the OsG1L2-containing subcluster C (Figure 4) might have a genetic interaction as predicted by the GRN.

A

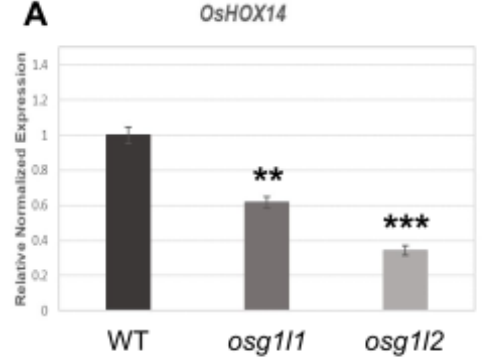

D

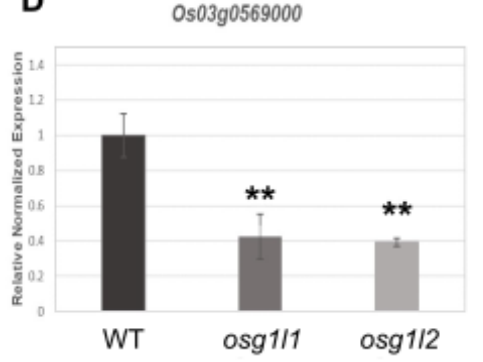

B

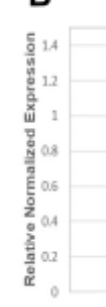

E
OSMADS34

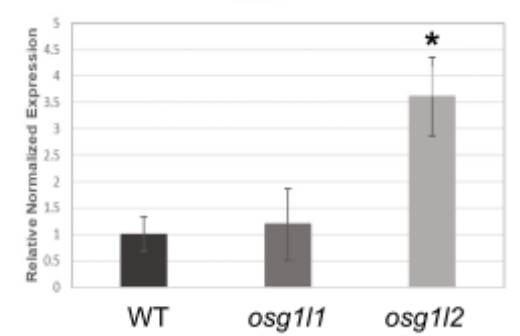

Figure 5. Expression analysis of subcluster $C$ genes in the osg1/1 and osg1/2 mutants.

Expression analysis of OsHOX14 (A), OsMADS34 (B), OsFC1 (C), Os03g0569000 (D), OsESP (E) by quantitative real-time PCR in wild type (WT), osg1/1 and osg1/2 mutants. Expression of OsHOX14, OsMADS34, OsFC1 and Os03g0569000 was normalized to that of Elongation Factor 1 and the expression level of wild type was set to 1 . The asterisks indicate: ${ }^{* * *} p<0,001 ;{ }^{* *} p<0,01$; * $p<0,05$, student's t-test.

\section{Oshox14 mutant exhibiting a branching phenotype}

The GRN subcluster $\mathrm{C}$, that contains OsG1L2, shows as major components OsMADS34, OsFC1 and OsHOX14. OsMADS34 and OsFC1 have been intensively studied for their role in inflorescence development (Takeda et al., 2003; Gao et al. 2010) but for OsHOX14 only an overexpression study has been reported (Shao et al., 2018). Therefore, we selected OsHOX14 for further functional studies to validate the GRN predicted involvement of this gene in inflorescence development.

OsHOX14 is a member of the homeodomain-leucine zipper (HD-Zip) transcription factor family and it is the rice orthologue of barley HvHox2 that, together with its recently diverged paralogue HvVrs 1 , are responsible for either the suppression or establishment of the barley lateral sterile spikelets 
382 (Sakuma et al., 2010). Indeed, OsHOX14, like OsG1L1 and OsG1L2 was shown to be expressed in 383 the inflorescence meristem tissues (PBM, ePBM/AM, SM) (Harrop et al., 2016). The spatiotemporal 384 expression of OsHOX14 throughout early panicle development was assessed with an in situ 385 hybridization experiment. This TF resulted to be expressed in PBM, ePBM/AM and SM/FM, 386 suggesting a putative role of this gene during reproductive meristems establishment (Figure 6 A-C). 387 We generated a knock-out mutant line for OsHOX14 using the CRISPR-Cas9 genome editing 388 system (Miao et al., 2013). A specific sgRNA was designed to target the first exon of the OsHOX14 389 gene (Supplemental Figure S4A). T0 transgenic plants were selected and genotyped. In the T1 390 generation, three different mutant lines were obtained, having respectively, a homozygous $\mathrm{G}$ 391 deletion, a homozygous $C$ insertion, and a homozygous CG deletion and T insertion at $7 \mathrm{bp}, 11 \mathrm{bp}$, 392 and 6 bp downstream the start site. In all three cases, the different mutations led to a frameshift in 393 the coding sequence and the formation of a premature stop codon, which resulted in the formation 394 of a protein consisting of 22, 27, and 22 amino acids, respectively (Supplemental Figure S4 B-C). 395 To evaluate the inflorescence phenotype of oshox14, a comparative analysis was performed on 396 panicles belonging to 5 wild-type and 5 T1 oshox 14 mutant plants (1 plant carrying the homozygous 397 C insertion mutation, 1 plant carrying the CG deletion and $T$ insertion mutation and 3 plants carrying 398 the $\mathrm{G}$ deletion mutation). This analysis revealed that the oshox14 mutants developed panicles with 399 less PBs and spikelets/seeds than wild-type plants. The number of SBs was not significantly different 400 from wild-type. In particular, the oshox14 mutant plants produced panicles that developed on 401 average 3 PBs and 20 spikelets less than the wild-type (Figure 6 D-G). Overall, this analysis shows 402 that OsHOX14 plays a role in inflorescence branching as predicted by the GRN. 

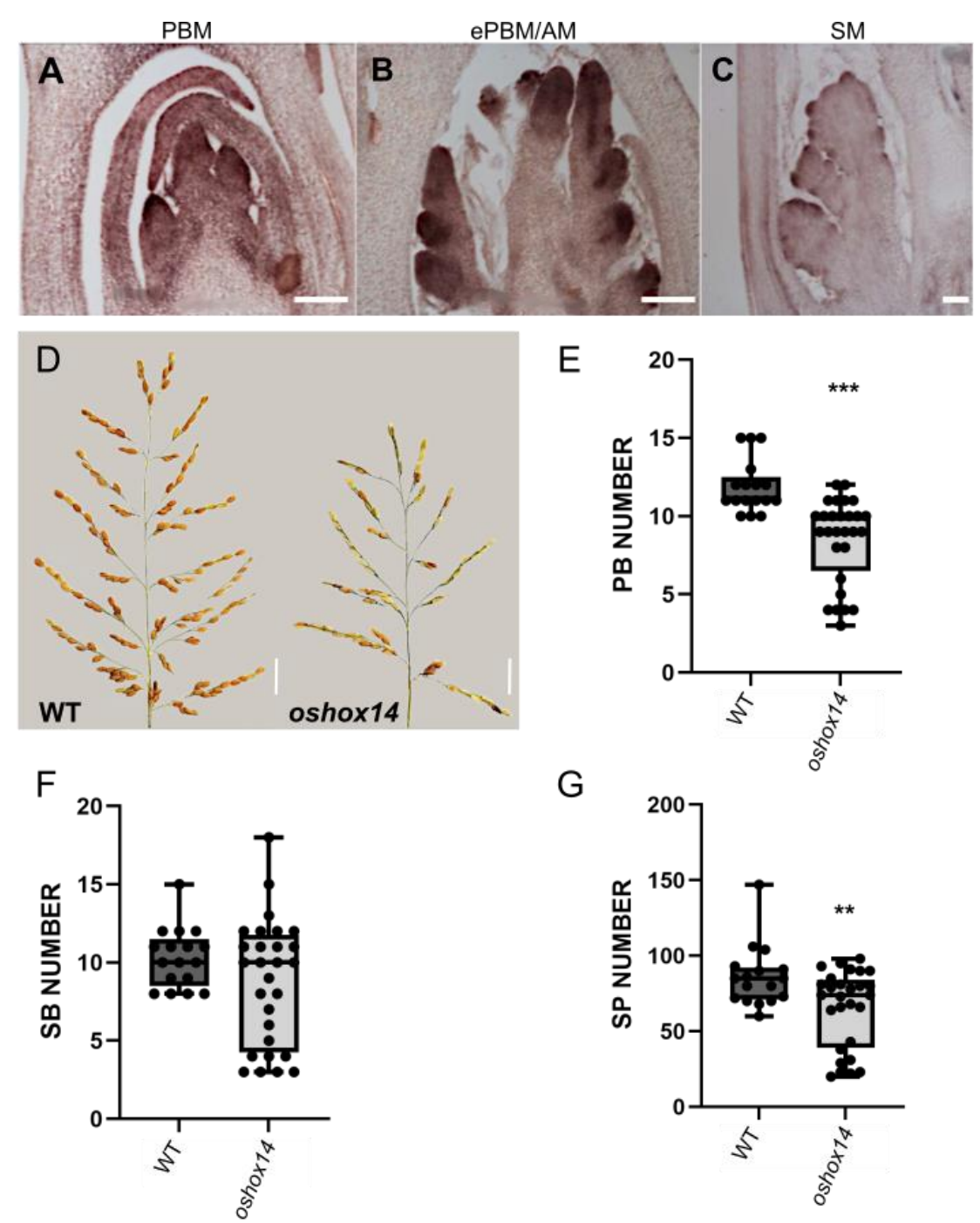

Figure 6. Expression and function analysis of OsHOX14. In situ analysis of three developmental stages: A, PBM; B, ePBM/AM and C, SM. Scale bars represent $50 \mu \mathrm{m}(\mathrm{C})$ and $100 \mu \mathrm{m}(\mathrm{A}-\mathrm{B})$. [Primary Branch Meristem (PBM), elongated PBM with Axillary Meristem (ePBM/AM), Spikelet Meristem (SM)]. D-G, Phenotypical analysis of panicle architecture in wild type and oshox14 mutant. $D$, main panicles of wild type (WT) and oshox14 $(2 \mathrm{~cm}$ scale bars). Graphs representing the comparison of: E, Primary Branch (PB) number; F, Secondary Branch (SB) number; G, Spikelet/seed (SP) number in WT and oshox14 backgrounds. ${ }^{* * *}=\mathrm{p}<0,001 ;{ }^{* *}=\mathrm{p}<0,01 ;{ }^{*}=\mathrm{p}<0,05$, Student's $\mathrm{t}-$ test.

\section{DISCUSSION}

415 Inflorescence architecture is a key agronomic trait that influences plant yield and its development is 416 finely regulated by genes involved in meristem identity specification and in the control of the transition 417 from indeterminate to determinate growth. Therefore, identifying genes involved in inflorescence 418 development promises to contribute to improved crop yield through breeding and biotechnological 419 approaches.

420 In this study, we functionally characterized OsG1L1 and OsG1L2, two rice genes belonging to the 421 ALOG gene family. These two genes are likely to play an important role in the regulation of 
inflorescence architecture specification acting as positive regulators of primary and secondary branch development. OsG1L1 and OsG1L2 were shown to be preferentially expressed in the reproductive meristems, in a pattern similar to TAW1, a gene already known to be involved in the development of the rice inflorescence by promoting indeterminate meristem identity (Yoshida et al 2013). Phenotypical analysis of the osg1/1 and osg1/2 mutant inflorescences showed that the two single mutants developed shorter panicles with fewer spikelets when compared to wild type. Furthermore, the mutant seeds were also shown to be smaller.

The observed aberrations in inflorescence architecture of the osg1/2 mutant and to less extent of the osg1/1 mutants indicate that they might be involved, like TAW1, in the regulation of the identity transition from BM to SM.

However, the DEG list obtained from our RNA-seq analysis of the osg1/2 mutant inflorescences did not include genes that were proposed to be acting downstream of TAW1 such as SVP family MADSbox genes or OsMADS7, OsMADS8, OsMADS16, OsMADS3 and OsMADS58 (Yoshida et al., 2013). This observation suggests that, as being part of two distinct phylogenetic groups, OsG1L2 (and probably also OsG1L1) may function in an inflorescence developmental pathway that acts in parallel with TAW1. However, further analysis will be needed to sustain this hypothesis since the DEG list published by Yoshida et al. (2013) was obtained from microarray studies using the taw1-D gain-of-function mutant whereas we used the osg1/2 knock-out mutant which showed an opposite phenotype.

The transcriptomic analysis of the osg1/2 reproductive meristems revealed that some of the differentially expressed genes are known to be involved in inflorescence architecture determination. For instance, in previous studies, OsRCN1 and OsRCN4 overexpression resulted in plants that produced panicles with an increased number of branches (Nakagawa et al., 2002; Liu et al., 2013) and knocking-down all RCN genes resulted in shorter panicles with fewer secondary branches (Liu et al., 2013). This phenotype is similar to the one observed in the osg1/2 mutant, where OsRCN1 and OsRCN4 are both downregulated. Furthermore, Nakagawa et al. (2002) indicate a role of $R C N$ genes in the suppression of spikelets meristem identity.

Another example is OsGATA7 which was downregulated in the osg1/2 mutant. Knock-down and knock-out mutants showed alterations in the architecture of the inflorescence. Knock-down of OsGATA7 results in panicles with a reduced number of primary branches, whereas the knock-out mutant develops panicles bearing fewer primary and secondary branches (Zhang et al., 2018). Based on the results obtained from our analyses it could be suggested that deregulation of OsRCN1,

454 OsRCN4 and OsGATA7 expression in the osg1/2 mutant might contribute to the observed 455 inflorescence phenotypes. Since in the predicted GRN, OsGATA7 resulted to be located in a different 456 subcluster than OsG1L2 (Figure 4), it is tempting to speculate that OsGATA7 regulates inflorescence 457 architecture in a parallel pathway. 
In the GRN proposed, PAP2/OsMADS34 falls in the same subcluster as OsG1L2. OsMADS34 belongs to the SEPALLATA (SEP) subfamily of the MADS-box gene family and is one of the key regulators of spikelet meristem identity in rice. However, for pap2/osmads34 mutants contrasting phenotypes have been published. Kobayashi et al. (2010) observed in the pap2-1 mutant a suppression of the transition of branch meristems into spikelet meristem identity resulting in more primary and secondary branches. While the osmads34 mutant described by Gao et al. (2010) showed also more primary branches but less secondary branches and spikelets. Both mutants had smaller panicles. Interestingly, in the osg1/2 mutant, OsMADS34 expression was reduced, which correlates with the observed reduced panicle length and the reduced number of SBs and spikelets observed in the osmads34 mutant described by Gao et al. (2010). However, the reduced number of PBs in the osg1/2 contrasts with the OsMADS34 downregulation. Overall, OsMADS34/PAP2 seems predominantly involved in promoting spikelet identity whereas OsG1L2 plays most likely an opposite role in this transition. Since OsMADS34 is still expressed in the osg1/2 mutant it is difficult to conclude which genetic interactions these genes may have. However, it is likely that their pathways are connected, and the balance of their activities may determine the final architecture of the panicle. The relationship between OsMADS34/PAP2 and OsG1L2 was furthermore predicted by the GRN that we developed in this study (Figure 4).

Some genes that were upregulated in the osg1/2 background had an expression level equal to zero in the wild type background. One of them is OsESP, which encodes for a long non-coding RNA (Luan et al., 2019). In the semi-dominant Epi-sp mutant, 3' region of the transcribed region was characterised by a loss of DNA methylation resulting in a strong upregulation of the gene causing the development of a denser and shorter panicle. It is possible that the reduction in panicle length observed in osg1/2 background is linked to the observed upregulation of ESP. It would be interesting to investigate whether the loss of OsG1L2 activity leads to changes in the 3' methylation of the ESP gene. ESP was one of the predicted direct targets of OsG1L2 within the GRN. The GRN also indicated that ESP (together with other 8 genes, still unknown and only expressed in osg $1 / 2$ mutant developing inflorescences) is a target of both OsG1L2 and OsFC1. It is known that OsFC1 controls panicle architecture since the null mutant developed smaller panicles (Cui et al.,2020). It is tempting to hypothesize that OsFC1 during the reproductive phase together with OsG1L2 represses ESP. Among the genes which we selected for real-time PCR validation, OsHOX14 showed a strong downregulation, especially in the osg1/2 mutant background. OsHOX14 is a member of the homeodomain-leucine zipper (HD-Zip) transcription factor family, and is known to play important roles in different aspects of plant development and morphogenesis and also in responses to biotic and abiotic stresses (Sessa et al., 2018). In particular, the results obtained in different species suggest a role of some HD-Zip I family proteins as integrators of internal and external signals in the regulation of abiotic and biotic stresses as well as in specific growth and developmental pathways 
the expression of OsHOX14 in reproductive meristems as reported by Harrop et al. (2016) and Shao et al. (2018). It is furthermore worth mentioning that the online RiceXPro tool (https://ricexpro.dna.affrc.go.jp/) showed the expression of OsHOX14 mainly in developing panicles and pistils, suggesting a specific role of this transcription factor during reproductive development. The CRISPR oshox14 mutants that we generated, confirmed a role for this gene in inflorescence development, since panicle development was impaired in these mutants, carrying fewer PBs and SPs when compared to wild-type plants. This phenotype is similar to the g1/2 mutant in which OsHOX14 expression was strongly reduced. The OsHOX14 overexpression lines generated by Shao et al. (2018) displayed dramatic phenotypes, such as severe delay in growth the seedling stage and difficulties with panicle exertion through stem and leaves. A low-overexpression line was analysed for panicle development which showed both a reduction in panicle length and PB number. This is coherent to our observations in the oshox14 knock-out CRISPR mutant. Since ectopic expression of OsHOX14 during the vegetative phase causes growth defects it might well be that this has pleiotropic effects on reproductive development. However, we cannot rule-out the possibility that the knock-out mutant caused a similar phenotype as the overexpression line because of the existence of regulatory loops and dependence on threshold levels that could influence regulatory pathways (Prelich, 2012).

Comparing the osg1/1 and osg1/2 CRISPR mutants revealed that the osg1/2 mutant rice plants present a more severe reduction of SBs. Furthermore, the osg1/2 panicles also present fewer spikelets than the osg1/1 single mutant. For these reasons, we hypothesize that OsG1L2 plays a more important role than OsG1L1 in inflorescence architecture development. Based on the RNAseq data, it is unlikely that OsG1L1 is regulated by OsG1L2, since OsG1L1 was not deregulated in the osg1/2 background. However, it is very well possible that OsG1L1 and OsG1L2 act in the same pathway. This hypothesis is further supported by our qRT-PCR studies that showed that the expression of some genes was deregulated in both mutants.

Overall, our analysis suggests that OsG1L1, OsG1L2 and TAW1/OsG1L5 seem to act in none or partially overlapping pathways. Furthermore, we propose for OsG1L2 and OsG1L1 a role in IM specification, branch formation, spikelets number determination and in seed development. Moreover, OsG1L2 seems to act in pathways that include OsMADS34, OsHOX14 and OsFC1. Finally, the functional analysis of OsHOX14 indicates that the proposed GRN promises to be of value

MATERIALS AND METHODS for the identification of new players in the first stages of inflorescence development. for $8-10$ weeks in LD conditions $\left(70 \%\right.$ humidity, $16 \mathrm{~h}$ light at $28^{\circ} \mathrm{C} / 8 \mathrm{~h}$ dark at $\left.26^{\circ} \mathrm{C}\right)$ and then moved in SD conditions $\left(70 \%\right.$ humidity, $12 \mathrm{~h}$ light at $28^{\circ} \mathrm{C} / 12 \mathrm{~h}$ dark at $\left.26^{\circ} \mathrm{C}\right)$ to induce flowering. In vitro, 
plants were germinated on MS-F medium (2,2 g/L MS + vitamins, $15 \mathrm{~g} / \mathrm{L}$ Sucrose, $1 \mathrm{~L} \mathrm{ddH2O}, \mathrm{pH}$ adjusted to 5.6 adding $\mathrm{KOH}, 2.5 \mathrm{~g} / \mathrm{L}$ gelrite) and after 15 days were transplanted in soil. Plants used for phenotypic analysis were grown in IRD transgenic greenhouse (Montpellier, France) in spring 2019 (seeds have been sown in February 2019 and panicles collected in June) under natural day conditions at $28^{\circ} \mathrm{C}-30^{\circ} \mathrm{C}$, and humidity at $60 \%$. Plants used for the RNAseq experiment were grown in the $\mathrm{C}$ chamber in NC State University Phytotron.

\section{RNA isolation and cDNA synthesis}

Total RNA from different tissues (roots, young and mature leaves, milk and mature seeds) and from meristematic tissue enriched in Inflorescence Meristems (IM), Primary Branch Meristems (PBMs), Elongated Primary branch meristems and Axillary Meristems (ePBM/AM) and Spikelet Meristems (SM) was extracted with the NucleoSpin® RNA Plant kit (http://www.mn-net.com) and DNA contamination was removed using the TURBO DNA-free ${ }^{\mathrm{TM}}$ Kit according to the manufacturer's instructions (https://www.thermofisher.com). The different tissues were sampled in liquid Nitrogen using an optical microscope. The RNA was reverse transcribed using the ImProm-II ${ }^{\mathrm{TM}}$ Reverse Transcription System (https://ita.promega.com) and the cDNA was used as a template in RT-PCR reactions.

\subsection{Transcriptome analysis}

80 plants (40 WT plants and 40 osg $1 / 2$ mutant plants) were sown in growth chamber under LD condition $\left(70 \%\right.$ humidity, $16 \mathrm{~h}$ light at $28^{\circ} \mathrm{C} / 8 \mathrm{~h}$ dark at $\left.26^{\circ} \mathrm{C}\right)$, at NC State University Phytotron and, after 12 day of induction in SD conditions, were sampled. Fifty mg of tissue, corresponding to 8-10 meristems at early developmental stages enriched in PBMs and ePBM/AMs, was manually dissected using an optical microscope. RNA was extracted from the samples using RNeasy Plant Mini Kit from Qiagene. cDNA libraries were prepared using NEBNext Ultra DNA Library Prep Kit for Illumina (E7370) according to the manufacturer's instructions. Novaseq6000 Illumina machine was used and sequencing was single-end stranded.

\subsection{Data analysis}

Raw RNA-seq data in fastq format of wild type and osg1/2 mutant was processed and subsequently used for gene regulatory network inference with the TuxNet interface (Spurney et al., 2019) (https://github.com/rspurney/TuxNet). For the processing of the raw RNA-seq, the gff and fasta file of the reference genome (IRGSP-1.0) and gene name file was downloaded from The Rice Annotation Project Database (RAP-DB; https://rapdb.dna.affrc.go.jp/download/irgsp1.html) (Supplemental Table S5). The gene IDs from rice transcription factors were downloaded from the plant transcription database v4.0 (http://planttfdb.gao-lab.org/) and converted from MSU to RAP , with the manual addition of the ALOG gene family. Next, TuxNet uses ea-utils fastq-mcf (Aronesty, 2013) for pre-processing, hisat2 (Kim et al., 2015) for genome alignment and Cufflinks (Trapnell et al., 2012) for differential expression analysis. The following xlsx files are generated by TuxNet: a file containing the FPKM values for each replicate (Supplemental Table S3), a file with the differentially expressed genes (DEGs) identified with a q-value threshold of 0.05 and a $\log _{2}$ (fold change) of 1 (Supplemental Table S4), and a file containing an average expression, a $\log _{2}$ (fold change), and a qvalue (Supplemental Table S6).

The PCA (principal component analysis) was performed in R with the RPKM file from TuxNet using the prcomp function from the stats package and the pca3d package (January Weiner (2020). pca3d: Three Dimensional PCA Plots. $R$ package version 0.10.2. https://CRAN.Rproject.org/package=pca3d) (Supplemental Figure S5). 
To infer the gene regulatory network (GRN) in osg1/2 and predict the causal relationships between and target genes underlying the inflorescence phenotype in osg 1/2, the differentially expressed TFs and genes identified in osg1/2 with a q-value threshold $<0.05$ and a $\log _{2}$ (fold change) $>1$ or $<-1$ (Supplemental Table S4) were selected. We manually added OsG1L2 to the DEGs list, since the $\log _{2} \mathrm{FC}$ of OsG1L2 was less than 1 (-0.885798). Within the TuxNet interface, RTP-STAR (Regression Tree Pipeline for Spatial, Temporal and Replicate data) leverages the replicate data of the wild type and osg1/2 and consists of three parts: spatial clustering using the k-means method, network inference using GENIE3 (regression tree with random forest approach) and edge sign (activation or repression) identification using the first-order Markov method. As options, we used 100 iterations when inferring the GRN and an edge proportion equal to 0.33 . The table containing the final predicted network (Supplemental Table S7) has been imported into Cytoscape® 3.8.0 (Shannon et al., 2003), a network visualization software, to obtain high-quality graphics representation of the predicted GRN. Different node shape, color, and size were used to represent TFs, the down- or upregulation in osg1/2, and the number of interactions, respectively. The nodes within the network were clustered into different modules with the Cytoscape plugin clusterMaker2 according to the available community clustering algorithm, an implementation of the Girvan-Newman fast greedy algorithm that uses connectivity to cluster nodes (Morris et al., 2011).

\section{4. qRT-PCR Analysis}

Fresh meristematic tissue enriched in Inflorescence Meristems (IM), Primary Branch Meristems (PBMs), Elongated Primary branch meristems and Axillary Meristems (ePBM/AM) and Spikelet Meristems (SM) was collected in liquid nitrogen using an optical microscope. RNA was extracted with the NucleoSpin® RNA Set for NucleoZOL - MACHEREY-NAGEL KIT for high purity products. The qRT-PCR analysis was carried out in a final volume of $12 \mu \mathrm{L}$ in a Biorad C1000 ${ }^{\mathrm{TM}}$ thermal cycler, using $3 \mu \mathrm{L}$ of a 1:10 dilution cDNA, 0,2 $\mu \mathrm{M}$ (stock 10mM) Forward and Reverse Primer, $6 \mu \mathrm{L}$ of Sybr Green Super Mix 2X (Bio-Rad), 2,6 $\mu \mathrm{MQ} \mathrm{M} 2 \mathrm{O}$.

The expression levels of OsG1L1(LOC_Os02g07030), OsG1L2 (LOC_Os06g46030) and TAW1 (LOC_Os10g33780) were evaluated using primer pairs RT2541/RT2542, RT1387/ RT1389 and RT2543/ RT2544 respectively. The RT-PCR was performed with the following conditions: $95^{\circ} \mathrm{C} 90^{\prime \prime}$ 40 cycles $\left(95^{\circ} \mathrm{C} 15^{\prime \prime}, 60^{\circ} \mathrm{C} 10^{\prime \prime}, 60^{\circ} \mathrm{C} 30^{\prime \prime}\right)$ and $60^{\circ} \mathrm{C} 10^{\prime \prime}$.

The expression levels of OsESP(Os01g0356951), OsMADS34/PAP2 (LOC_Os03g54170), OsHOX14 (LOC_Os07g39320), OsCEP6 (LOC_Os08g37070.1), OsTB1/FC1 (LOC_Os03g49880) and Os03g0569000 in WT and osg1/2 background were evaluated using primer pairs OSP2055/OSP2056, OSP0855/OSP0856, OSP1400/1401, OSP2043/OSP2044, OSP2045/OSP2046, OSP2059/OSP2060 and using the following condition: $95^{\circ} \mathrm{C} 90^{\prime \prime} 40$ cycles $\left(95^{\circ} \mathrm{C} 15^{\prime \prime}, 58^{\circ} \mathrm{C} 10^{\prime \prime}, 60^{\circ} \mathrm{C} 30^{\prime \prime}\right)$ and $60^{\circ} \mathrm{C} 10^{\prime \prime}$.

Three biological replicates for each experiment were performed.

Rice Elongation Factor 1 (EF1) (LOC_Os03g08010) was used as an internal reference during the experiments. Primer sequences are listed in Supplemental Table S8.

\section{Tissue fixation and In situ Hybridization}

Rice reproductive meristems from the main stem at different stages of early panicle development were collected and fixed in FAA [ethanol (Fluka) $50 \%$; acetic acid (Sigma-Aldrich) $5 \%$; formaldehyde (Sigma-Aldrich) $3.7 \%(\mathrm{v} / \mathrm{v})$ ], infiltrated under mild vacuum conditions for $15 \mathrm{~min}$ in ice. After $1 \mathrm{~h} 45^{\prime}$ the samples were washed 3 times for $10^{\prime}$ in $\mathrm{EtOH} 70 \%$ and conserved at $4^{\circ} \mathrm{C}$; they were dehydrated in a series of increasing graded ethanol series, transferred to bioclear (Bioptica) and then embedded in Paraplast X-TRA® (Sigma-Aldrich). To generate the sense and antisense probes, gene fragments were amplified from cDNA using gene-specific primers (Supplemental Table S8), cloned into PGEM®-T Easy Vector and confirmed by sequencing. Digoxigenin-labeled antisense and 
sense RNA probes were transcribed and labelled from pGEM ${ }^{\circledR}-$ T Easy $^{-}$with T7/SP6 RNA polymerase (Promega) according to the manufacturer's instructions and using the DIG RNA labelling mix (Roche). Paraplast-embedded tissues were sliced on an RM2155 microtome (Leica) at $8 \mu \mathrm{m}$ of thickness and hybridized as described by Caselli et al. (2019) with minor modifications. Immunodetection was carried out with anti-digoxigenin-AP Fab fragment (Roche) and BCIP-NBT colour development substrate (Promega) as specified by the manufacturer. Sample's images were acquired with a Zeiss Axiophot D1 (Zeiss, Oberkochen, Germany) microscope with an Axiocam MRc 5 (Zeiss) at different magnifications.

\section{CRISPR-Cas9 construct generation}

For the generation of osg1/1, osg1/2 and oshox14 (LOC_Os07g39320) single knock-out mutants, 20-bp specific protospacers (Supplemental Table S8) for each gene were selected using the CRISPR-P database (http://cbi.hzau.edu.cn/crispr/) and cloned into the Bsal site of pOs-sgRNA entry vectors under U3 promoter and then combined into the destination vector containing the Cas9 under maize Ubiquitin Promoter using the Gateway® LR Clonase II Enzyme mix following the procedure reported by Miao et al. (2013) and already followed by Lacchini et al., 2020.

\section{Bacterial and plant transformation}

For bacterial transformation, we used $E$. coli electrocompetent cell (DH10b strains) and Agrobacterium tumefaciens electrocompetent cell (EH105 strain).

All final constructs were used to transform embryogenic calli obtained from Oryza sativa L. ssp. japonica cv. Nipponbare seeds according to the methods described by Hiei et al. (1994) and Toki

\section{Mutant screening in transgenic plants}

Genomic DNA was extracted from T0-hygromycin-resistant rice plants and genotyped by PCR using primers specific for Cas9 construct, Atp5706/Atp5718 (Supplemental Table S8). Subsequently, from the positive plants, DNA fragments across the target sites were amplified through PCR using genespecific primer pairs (Supplemental Table S8). The PCR amplicons were purified and sequenced. The obtained chromatograms were analysed and compared with WT sequences with FinchTV searching for mutations.

\section{Phenotypical analysis of panicles and seeds}

To perform phenotypical analysis 15, 19 and 20 panicles from the main tiller were collected respectively from WT, osg1/1 and osg1/2 plants. Each panicle was attached on A4 white paper and all panicle branches were spread and blocked with transparent sticks. Each paper with panicle and scale bar was put on an Image capturing system consisting of Portable Camera Stand and two RB 218N HF Lighting Units. The pictures were processed into P-TRAP software. The analysis was done as described in AL-Tam et al., (2013). The results were statistically analysed by One Way ANOVA followed by Tukey test and represented with GraphPad Prism 8.

To perform the phenotypical analysis on wild type and oshox14 plants, all the panicles produced by 5 wild-type and 5 oshox 14 plants were collected. For each panicle, the number of Primary Branches (PBs), Secondary Branches (SBs) and Spikelets/seeds (SP) was manually calculated. The results were statistically analysed with a Student's T-test and graphically represented with GraphPad Prism 8.

At least one hundred seeds were analysed for each genotype (osg1/1, osg1/2 and WT). Images of the seeds were acquired using a Leica MZ6 stereomicroscope in conjunction with a Leica DFC280 camera at different magnifications; each image was then processed with Smart Grain (Tanabata et 
al. 2012). The obtained results were statistically analysed with a One Way ANOVA followed by Tukey test and represented with GraphPad Prism 8.

\section{Accession Numbers:}

Sequence from this article can be found in the GeneBank / EMBL databases under the following accession numbers: OsG1L1 LOC_Os02g07030, OsG1L2 LOC_Os06g46030, TAW1 LOC_Os10g33780, OsESP Os01g0356951, OsMADS34/PAP2 LOC_Os03g54170, OsHOX14 LOC_Os07g39320, OsCEP6 LOC_Os08g37070.1, OsTB1/FC1 LOC_Os03g49880,

\section{Supplemental Data}

The following supplemental materials are available:

Supplemental Figure $\mathbf{S 1}$ Gene structure and wild type and mutant proteins alignment of OsG1L1. Supplemental Figure S2 Gene structure and wild type and mutant proteins alignment of OsG1L2. Supplemental Figure S3 Chromatogram showing the type of mutation and phenotypical analysis of osg1/2 mutant (C insertion).

Supplemental Figure S4 Gene structure and wild type and mutant proteins alignment of OsHOX14. Supplemental Figure S5 PCA output.

Supplemental Table S1 Phenotypical traits analysed in wild type, osg1/1 and osg1/2 plants.

Supplemental Table S2 Area, length and width of wild type, osg1/1 and osg1/2 seeds.

Supplemental Table S3 FPKM values for each replica.

Supplemental Table S4 Differentially expressed genes between wild type and osg 112 mutants. Supplemental Table S5 List of gene names.

Supplemental Table S6 Average gene expression, $\log _{2}$ (fold change) and q-value in wild type and osg1/2 mutant.

Supplemental Table S7 Final predicted GRN.

Supplemental Table S8 Primers used in this article.

\section{ACKNOWLEDGMENTS}

We like to thank Andrea Guazzotti and Marco Maffei for their help with the analysis of the oshox14 mutant. Furthermore, we thank Mario Beretta and Valerio Parravicini for taking care of the rice plants.

\section{REFERENCES}

A L-Tam, F., Adam, H., Anjos, A. dos, Lorieux, M., Larmande, P., Ghesquière, A., ... Shahbazkia, 
H. R. (2013). P-TRAP: a Panicle TRAit Phenotyping tool. BMC Plant Biology, 13, 122. https://doi.org/10.1186/1471-2229-13-122

Aronesty, E. (2013). Comparison of Sequencing Utility Programs. The Open Bioinformatics Journal, 7(1), 1-8. https://doi.org/10.2174/1875036201307010001

Bommert, P., Satoh-Nagasawa, N., Jackson, D., Hirano, HY., (2005) Genetic and evolution of grass inflorescence and flower development in grasses. Plant and Cell Physiology 46, 6978, https://doi.org/10.1093/pcp/pci504

Caselli, F., Zanarello, F., Kater, M. M., Battaglia, R., \& Gregis, V. (2020). Crop reproductive meristems in the genomic era: A brief overview. Biochemical Society Transactions, 48(3), 853865. https://doi.org/10.1042/BST20190441

Cheng, X., Jiang, H., Zhang, J., Qian, Y., Zhu, S., \& Cheng, B. (2010). Overexpression of type-A rice response regulators, OsRR3 and OsRR5, results in lower sensitivity to cytokinins. Genetics and Molecular Research: GMR, 9(1), 348-359. https://doi.org/10.4238/vol9-1gmr739

Cui, Y., Hu, X., Liang, G., Feng, A., Wang, F., Ruan, S., .. Qian, Q. (2020). Production of novel beneficial alleles of a rice yield-related QTL by CRISPR/Cas9. Plant Biotechnology Journal, 18(10), 1987-1989. https://doi.org/10.1111/pbi.13370

Gao, X., Liang, W., Yin, C., Ji, S., Wang, H., Su, X., ... Zhang, D. (2010). The SEPALLATA-like gene OsMADS34 is required for rice inflorescence and spikelet development. Plant Physiology, 153(2), 728-740. https://doi.org/10.1104/pp.110.156711

Gao, S., Fang, J., Xu, F., Wang, W., \& Chu, C. (2016). Rice HOX12 regulates panicle exsertion by directly modulating the expression of ELONGATED UPPERMOST INTERNODE1. Plant Cell, 28(3), 680-695. https://doi.org/10.1105/tpc.15.01021

Hakata, M., Kuroda, M., Ohsumi, A., Hirose, T., Nakamura, H., Muramatsu, M., ... Yamakawa, H. (2012). Overexpression of a rice tify gene increases grain size through enhanced accumulation of carbohydrates in the stem. Bioscience, Biotechnology and Biochemistry, 76(11), 2129-2134. https://doi.org/10.1271/bbb.120545

Hake, S. (2008). Inflorescence architecture: the transition from branches to flowers, Current Biology, Volume 18, Issue 23, R1106-R1108, https://doi.org/10.1016/j.cub.2008.10.024.

Han, Y., Yang, H., \& Jiao, Y. (2014). Regulation of inflorescence architecture by cytokinins. Frontiers in Plant Science, 5(November), 669. https://doi.org/10.3389/fpls.2014.00669

Harrop, T. W. R., Ud Din, I., Gregis, V., Osnato, M., Jouannic, S., Adam, H., \& Kater, M. M. (2016). Gene expression profiling of reproductive meristem types in early rice inflorescences by laser microdissection. Plant Journal, 86(1), 75-88. https://doi.org/10.1111/tpj.13147

Hiei, Y., Ohta, S., Komari, T., \& Kumashiro, T. (1994). Efficient transformation of rice (Oryza sativa L.) mediated by Agrobacterium and sequence analysis of the boundaries of the T- DNA. The Plant Journal, 6(2), 271-282. https://doi.org/10.1046/j.1365-313X.1994.6020271.x

Huang, L., Hua, K., Xu, R., Zeng, D., Wang, R., Dong, G., ... Li, Y. (2021). The LARGE2-APO1/APO2 regulatory module controls panicle size and grain number in rice. The Plant Cell, 1-17. https://doi.org/10.1093/plcell/koab041

Hudson, D., Guevara, D., Hand, A., Xu, Z., Hao, L., Chen, X., Zhu, T., Bi, Y., Rothstein, S. (2013). Rice cytokinin GATA transcription Factor1 regulates chloroplast development and plant architecture. Plant Physiology PMID: 23548780 PMCID: PMC3641198 DOI: 10.1104/pp.113.217265

Ikeda-Kawakatsu, K., Maekawa, M., Izawa, T., Itoh, J. I., \& Nagato, Y. (2012). ABERRANT PANICLE ORGANIZATION 2/RFL, the rice ortholog of Arabidopsis LEAFY, suppresses the transition from inflorescence meristem to floral meristem through interaction with APO1. Plant Journal, 69(1), 168-180. https://doi.org/10.1111/j.1365-313X.2011.04781.x

Ikeda-Kawakatsu, K., Yasuno, N., Oikawa, T., lida, S., Nagato, Y., Maekawa, M., \& Kyozuka, J. (2009). Expression level of ABERRANT PANICLE ORGANIZATION1 determines rice 
inflorescence form through control of cell proliferation in the meristem. Plant Physiology, 150(2), 736-747. https://doi.org/10.1104/pp.109.136739

lyer, L. M., \& Aravind, L. (2012). ALOG domains: Provenance of plant homeotic and developmental regulators from the DNA-binding domain of a novel class of DIRS1-type retroposons. Biology Direct, 7(1), 1. https://doi.org/10.1186/1745-6150-7-39

Jain, M., Kaur, N., Garg, R., Thakur, J. K., Tyagi, A. K., \& Khurana, J. P. (2006). Structure and expression analysis of early auxin-responsive Aux/IAA gene family in rice (Oryza sativa). Functional and Integrative Genomics, 6(1), 47-59. https://doi.org/10.1007/s10142-005-0005-0

Kim, D., Langmead, B., \& Salzberg, S. L. (2015). HISAT: A fast spliced aligner with low memory requirements. Nature Methods, 12(4), 357-360. https://doi.org/10.1038/nmeth.3317

Kobayashi, K., Yasuno, N., Sato, Y., Yoda, M., Yamazaki, R., Kimizu, M., ... Kyozukaa, J. (2012). Inflorescence meristem identity in rice is specified by overlapping functions of three AP1/FULLike MADS box genes and PAP2, a SEPALLATA MADS Box gene. Plant Cell, 24(5), 18481859. https://doi.org/10.1105/tpc. 112.097105

Lacchini, E., Kiegle, E., Castellani, M., Adam, H., Jouannic, S., Gregis, V., \& Kater, M. M. (2020). CRISPR-mediated accelerated domestication of African rice landraces. PLoS ONE, 15(3), 112. https://doi.org/10.1371/journal.pone.0229782

Li, N., Wang, Y., Lu, J., \& Liu, C. (2019). Genome-wide identification and characterization of the ALOG domain genes in rice. International Journal of Genomics, 2019. https://doi.org/10.1155/2019/2146391

Li, Y. S., Sun, H., Wang, Z. F., Duan, M., Huang, S. D., Yang, J., ... Zhang, H. S. (2013). A novel nuclear protein phosphatase $2 \mathrm{C}$ negatively regulated by $\mathrm{ABL} 1$ is involved in abiotic stress and panicle development in rice. Molecular Biotechnology, 54(2), 703-710. https://doi.org/10.1007/s12033-012-9614-8

Liu, C., Teo, Z. W. N., Bi, Y., Song, S., Xi, W., Yang, X., ... Yu, H. (2013). A conserved genetic pathway determines inflorescence architecture in Arabidopsis and rice. Developmental Cell, 24(6), 612-622. https://doi.org/10.1016/j.devcel.2013.02.013

Luan, X., Liu, S., Ke, S., Dai, H., Xie, X. M., Hsieh, T. F., \& Zhang, X. Q. (2019). Epigenetic modification of ESP, encoding a putative long noncoding RNA, affects panicle architecture in rice. Rice, 12(1), 20. https://doi.org/10.1186/s12284-019-0282-1

Miao, J., Guo, D., Zhang, J., Huang, Q., Qin, G., Zhang, X., ... Qu, L.-J. (2013). Targeted mutagenesis in rice using CRISPR-Cas system. Cell Research, 23(10), 1233-1236. https://doi.org/10.1038/cr.2013.123

Morris, J. H., Apeltsin, L., Newman, A. M., Baumbach, J., Wittkop, T., Su, G., ... Ferrin, T. E. (2011). ClusterMaker: A multi-algorithm clustering plugin for Cytoscape. BMC Bioinformatics, 12, 1-14. https://doi.org/10.1186/1471-2105-12-436.

Nakagawa, M., Shimamoto, K., \& Kyozuka, J. (2002). Overexpression of RCN1 and RCN2, rice TERMINAL FLOWER 1 CENTRORADIALIS homologs, confers delay of phase. 29.

Nakano, T., Suzuki, K., Fujimura, T., \& Shinshi, H. (2006). Genome-wide analysis of the ERF gene family in arabidopsis and rice. Plant Physiology, 140(2), 411-432. https://doi.org/10.1104/pp.105.073783

Ooka, H., Satoh, K., Doi, K., Nagata, T., Otomo, Y., Murakami, K., ... Kikuchi, S. (2003). Comprehensive Analysis of NAC Family Genes in Oryza sativa and Arabidopsis thaliana. DNA Research, 10(6), 239-247. https://doi.org/10.1093/dnares/10.6.239

Perotti, M. F., Ribone, P. A., \& Chan, R. L. (2017). Plant transcription factors from the homeodomainleucine zipper family I. Role in development and stress responses. IUBMB Life, 69(5), 280 289. https://doi.org/10.1002/iub.1619

Prelich, G. (2012). Gene overexpression: Uses, mechanisms, and interpretation. Genetics, 190(3), 
841-854. https://doi.org/10.1534/genetics.111.136911

Ruelens, P., De Maagd, R. A., Proost, S., Theißen, G., Geuten, K., \& Kaufmann, K. (2013). FLOWERING LOCUS $\mathrm{C}$ in monocots and the tandem origin of angiosperm-specific MADS-box genes. Nature Communications, 4. https://doi.org/10.1038/ncomms3280

Sakuma, S., Pourkheirandish, M., Matsumoto, T., Koba, T., \& Komatsuda, T. (2010). Duplication of a well-conserved homeodomain-leucine zipper transcription factor gene in barley generates a copy with more specific functions. Functional and Integrative Genomics, 10(1), 123-133. https://doi.org/10.1007/s10142-009-0134-y

Sessa, G., Carabelli, M., Possenti, M., Morelli, G., \& Ruberti, I. (2018). Multiple links between HDZip proteins and hormone networks. International Journal of Molecular Sciences, 19(12). https://doi.org/10.3390/ijms19124047

Shannon, P., Markiel, A.,Ozier, O., Baliga, N., Wang, J., Ramage, D., Amin, N., Schwikowski, B., Ideker T. (2003). Cytoscape: A Software Environment for Integrated Models. Genome Research, 13(22), 426. https://doi.org/10.1101/gr.1239303.metabolite

Shao, J., Haider, I., Xiong, L., Zhu, X., Hussain, R. M. F., Övernäs, E., ... Ouwerkerk, P. B. F. (2018). Functional analysis of the HD-Zip transcription factor genes Oshox12 and Oshox14 in rice. PLOS ONE, 13(7), e0199248. https://doi.org/10.1371/journal.pone.0199248

Shrestha, R., Gómez-Ariza, J., Brambilla, V., \& Fornara, F. (2014). Molecular control of seasonal flowering in rice, arabidopsis and temperate cereals. Annals of Botany, 114(7), 1445-1458. https://doi.org/10.1093/aob/mcu032

Spurney, R. J., Van den Broeck, L., Clark, N. M., Fisher, A. P., de Luis Balaguer, M. A., \& Sozzani, R. (2019). tuxnet: a simple interface to process RNA sequencing data and infer gene regulatory networks. In Plant Journal (Vol. 101). https://doi.org/10.1111/tpj.14558

Sui, Z., Wang, T., Li, H., Zhang, M., Li, Y., Xu, R., ... Xin, M. (2016). Overexpression of peptideencoding OsCEP6.1 results in pleiotropic effects on growth in rice (O. sativa). Frontiers in Plant Science, 7(MAR2016), 1-12. https://doi.org/10.3389/fpls.2016.00228

Takeda, T., Suwa, Y., Suzuki, M., Kitano, H., Ueguchi-Tanaka, M., Ashikari, M., ... Ueguchi, C. (2003). The OsTB1 gene negatively regulates lateral branching in rice. The Plant Journal : For Cell and Molecular Biology, 33(3), 513-520. https://doi.org/10.1046/j.1365-313x.2003.01648.x

Tanabata, T., Shibaya, T., Hori, K., Ebana, K., Yano, M., (2012). SmartGrain: High-Throughput Phenotyping Software for Measuring Seed Shape through Image Analysis. Plant Physiology, 160 (4) 1871-1880; https://doi.org/10.1104/pp.112.205120

Tanaka, W., Pautler, M., Jackson, D., \& Hirano, H.-Y. (2013). Grass Meristems II: Inflorescence Architecture, Flower Development and Meristem Fate. Plant and Cell Physiology, 54(3), 313324. https://doi.org/10.1093/pcp/pct016

Toki, S. (1997). Rapid and efficient Agrobacterium-mediated transformation in rice. Plant Molecular Biology Reporter, 15(1), 16-21. https://doi.org/10.1007/BF02772109

Trapnell, C., Roberts, A., Goff, L., Pertea, G., Kim, D., Kelley, D. R., .. Pachter, L. (2012). Differential gene and transcript expression analysis of RNA-seq experiments with TopHat and Cufflinks. Nature Protocols, 7(3), 562-578. https://doi.org/10.1038/nprot.2012.016

Van Ooijen, G., Mayr, G., Kasiem, M. M. A., Albrecht, M., Cornelissen, B. J. C., \& Takken, F. L. W. (2008). Structure-function analysis of the NB-ARC domain of plant disease resistance proteins. Journal of Experimental Botany, 59(6), 1383-1397. https://doi.org/10.1093/jxb/ern045

Wang, J., Zhang, Q., Wang, Y., Huang, J., Luo, N., Wei, S., \& Jin, J. (2019). Analysing the rice young panicle transcriptome reveals the gene regulatory network controlled by TRIANGULAR HULL1. Rice, 12(1), 6. https://doi.org/10.1186/s12284-019-0265-2

Wu, K. L., Guo, Z. J., Wang, H. H., \& Li, J. (2005). The WRKY family of transcription factors in rice and arabidopsis and their origins. DNA Research, 12(1), 9-26. 
https://doi.org/10.1093/dnares/12.1.9

Yoshida, A., Sasao, M., Yasuno, N., Takagi, K., Daimon, Y., Chen, R., ... Kyozuka, J. (2013). TAWAWA1, a regulator of rice inflorescence architecture, functions through the suppression of meristem phase transition. Proceedings of the National Academy of Sciences of the United States of America, 110(2), 767-772. https://doi.org/10.1073/pnas.1216151110 OsGATA7 modulates brassinosteroids-mediated growth regulation and influences architecture and grain shape. Plant Biotechnology Journal, Vol. 16, pp. 1261-1264. 